REICE

Revista Electrónica de Investigación en Ciencias Económicas

Abriendo Camino al Conocimiento

Vol. 5, No. 10, julio - diciembre 2017

REICE ISSN: 2308-782X

REICE | 115

http://revistacienciaseconomicas.unan.edu.ni/index.php/REICE

revistacienciaseconomicas@gmail.com

\title{
Estimación del Balance Fiscal Estructural en Nicaragua Para el Periodo 1994 - 2016
}

\section{Estimation of the structural Fiscal Balance in Nicaragua for the period 1994-2016}

Fecha recepción: noviembre 12 del 2017

Fecha aceptación: noviembre 25 del 2017

Oliver David Morales Rivas

ID Orcid: https://orcid.org/0000-0002-9997-8679

Universidad Nacional Autónoma de Nicaragua, Managua

Departamento de Economía

oliverdavid19@gmail.com

Leonel Antonio Flores Mendez

ID Orcid: https://orcid.org/0000-0002-3416-5044

Universidad Nacional Autónoma de Nicaragua, Managua

Departamento de Economía

leonelflores088@gmail.com 


\title{
Resumen
}

El artículo presenta la estimación de un indicador de balance fiscal estructural para Nicaragua. Para ello, se utilizó la metodología general propuesta por FMI, para lograr este propósito estimaron dos modelos econométricos, el primero para determinar la elasticidad de los ingresos tributarios y el segundo para la elasticidad gasto corriente con respecto al Producto Interno Bruto Nacional Real y también se usó el Filtro Hodrick- Prescott para estimar el PIB Potencial, los datos se obtuvieron del Anuario Estadístico del Banco Central de Nicaragua. El balance fiscal estructural estimado empíricamente tiene el mismo comportamiento que la brecha entre producto efectivo y el potencial, además se infiere que el resultado de la política fiscal obedece a un componente estructural de la actividad económica.

Palabras clave: Ingreso, Gasto, Balance fiscal estructural, PIB Potencial, Política Fiscal.

\begin{abstract}
The article presents the estimation of a structural fiscal balance indicator for Nicaragua. For this, the general methodology proposed by IMF was used, to achieve this purpose they estimated two econometric models, the first to determine the elasticity of tax revenues and the second for the current expenditure elasticity with respect to the Real National Gross Domestic Product and also used the Hodrick-Prescott filter to estimate Potential GDP, the data were obtained from the Statistical Yearbook of the Central Bank of Nicaragua. The structural fiscal balance estimated empirically has the same behavior as the gap between effective product and potential, in addition it is inferred that the result of fiscal policy obeys to a structural component of economic activity.
\end{abstract}

Keywords: Public Income, Public spending, Fiscal Balance,PIB GDP Potential, Deficit, Fiscal policy. 


\section{Introducción}

Uno de los principales problemas que presenta la macroeconomía, es el efecto cíclico del crecimiento de la producción. Al realizar observaciones en un periodo determinado al desenvolvimiento del Producto Interno Bruto (PIB), queda de manifiesto si auges y sus depresiones a los largo del tiempo. Estos efectos cíclicos están directamente relaciones a la toma de decisiones de las políticas económicas a ejecutarse por los gobiernos, con las llamadas políticas anti-cíclicas cuando estas son negativas o tendencias a una disminución del ritmo de crecimiento (crisis).

La política fiscal es la principal que es utilizada para efecto que contrarrestar un hecho negativo en el crecimiento de la producción, esta política puede ser empleada desde el ajuste en la asignación del Gasto Publico o en la reforma de aplicación de impuesto, sin embargo (Price, 2011) afirma que:

El indicador los saldos presupuestarios tradicionales ajustados cíclicamente, que se utilizan para medir los saldos estructural o subyacente, no se ajusta a los efectos de las fluctuaciones cíclicas en los precios de los activos. Como resultado, de forma predeterminada, los efectos de los precios de los activos en los ingresos se tienen en cuenta en la medición del presupuesto estructural. Esto puede inducir a error a los responsables de la toma de decisiones públicas en caso de variaciones temporales precios de los activos, lo que lleva a una acción fiscal pro-cíclica, especialmente cuando las medidas de alivio son los impuestos o el aumento de los gastos se toman en presencia de una abundancia inesperada de ingresos. (pág. 2)

La importancia de la investigación radica en lo antes mencionada. Realizar un estudio del balance estructural resulta un indicador bastante eficiente para detectar operaciones discrecionales de políticas fiscales y cambios derivados de los ciclos económicas. 
En este mismo sentido, la identificación de acciones discrecionales en la realización de políticas públicas, resulta imprescindible para poder incorporar metas fiscales a alcanzar en el mediano y el largo plazo, sin embargo, para poder realizar esto principalmente "es necesario descomponer el ingreso y el gasto de sus componentes cíclicos y de los estructurales. Para esto se estiman elasticidades-producto de los componentes del ingreso y del gasto, lo que permite descomponer los elementos cíclicos y estructurales" (Beteta, 2012)(pag.6).

Los efectos cíclicos del crecimiento económico pueden ser atribuidos a condiciones endógenas y exógenas. Esta segunda es considerada todo choque externo que afecte al buen desenvolvimiento de la economía nacional. Por ejemplo la incertidumbre de la aprobación de la ley Nicaraguan Investmente Conditionality Act of 2017 o la propuesta de disminución del Impuesto sobre la Renta en Estados Unidos de América (EUA) que se ejecuta para el próximo año 2018. Desde este segundo punto, se debe de recordar que Nicaragua tiene como una de sus principales estrategias la atracción de Inversión Extranjera Directa (IED) para seguir con el ritmo de crecimiento, sin embargo, la disminución de 15 puntos porcentuales del Impuesto sobre la Renta Corporativa en EUA, de 35\% a $20 \%$, va a provocar que las empresas que residen en territorio nacional podrán reinvertir o enviar sus capitales a las casas matrices, provocando un flujo de capital de Nicaragua a EUA. 


\section{Material y Métodos}

En este acápite se presenta la metodología utilizada para determinación del balance fiscal estructural en Nicaragua, este artículo es eminentemente REICE | 119 cuantitativo, para su determinación se calculó el PIB Potencial, adicional se estimaron dos modelos econométricos y finalmente se empleó la metodología establecida por Fondo Monetario Internacional(FMI) para la determinación del balance fiscal estructural. A continuación, se detalla el procedimiento de cálculo.

Para la estimación del PIB Potencial en Nicaragua se utilizó el filtro univariante Hodrick - Prescott (FHP de acá en adelante), como menciona (Segura Rodríguez \& Vásquez Carvajal, 2011) el FHP consiste determinar valores más suavizados a los registrados realmente en una serie de tiempo, con esto se logra obtener una tendencia alrededor de la cual fluctúa la serie observado, para este caso el Producto Interno Bruto (PIB). La metodología del Filtro Hodrick - Prescott, minimiza la varianza del producto $Y$ en torno a su valor de tendencia $Y^{\prime}$, sujeto a una restricción sobre $Y^{\prime}$. La especificación matemática es la siguiente.

$$
\min \sum_{\mathrm{t}-1}^{\mathrm{T}}\left(Y_{t}-Y_{t}^{\prime}\right)^{2}+\lambda \sum_{\mathrm{t}-2}^{t-1}\left[\left(Y_{t+1}^{\prime}-Y_{t}^{\prime}\right)-\left(Y_{t}^{\prime}-Y_{t-1}^{\prime}\right)\right]^{2}
$$

donde $\lambda$ es un parámetro que determina la magnitud de suavización. Cuando más alto es el valor $\lambda$, más suavizada es la serie. La elección del valor $\lambda$ según (Segura Rodríguez \& Vásquez Carvajal, 2011), depende de los datos usados por el investigador, es así, que para datos anuales, Hodrick y Prescott recomiendan un valor de $\lambda=100$ y para datos trimestrales de $\lambda=1600$. En esta investigación se usó el FHP con datos anuales y un parámetro $\lambda=100$.

En esta investigación el PIB Potencial es el valor suavizado con el filtro Hodrick Prescott de la variable PIB real de Nicaragua a precios de referencia de 2006, la estimación se efectuó en el programa de econometría Eviews 9. 
La metodología utilizada en esta investigación para la determinación del balance fiscal estructural en Nicaragua, es la propuesta por el Fondo Monetario Internacional, a partir de la propuesta de (Hagémann, 1999). Es autor parte de la premisa de definición de balance corriente del sector público, es decir, de la diferencia de ingreso y gasto en un año determinado.

$$
\{2\} \quad B_{t}=I R T_{t}-G t
$$

El elemento fundamental de esta metodología es que separa los componentes estructurales y cíclicos de las variables gasto corriente e ingreso tributario.

$$
\begin{gathered}
\{3\} \quad B_{t}=B^{C}+B^{S} \\
\{4\} I R T_{t}=I R T^{C}+R^{S} \\
\{5\} \quad G_{t}=G^{C}+G^{S}
\end{gathered}
$$

Donde $B_{t}$ el balance fiscal que es el resultado de la diferencia de los ingresos tributarios $\left(R_{t}\right)$ y del gasto corriente del gobierno central $\left(G_{t}\right)$. Los subíndices c y s representa el componente cíclico y estructural respectivamente, la ecuación de balance estructural fiscal se puede organizar en :

$$
\{6\} \quad B_{t}=\left(I R T_{C}+I R T_{S}\right)-\left(G_{C}+G_{S}\right)
$$

Para el cálculo del componente estructural de los ingresos fiscales, se establece como supuesto base que los ingresos del Gobierno que se ven afectados por desempeño de la actividad económica (ciclo económico) y otros factores exógenos que se presentan de manera independiente y no están incididos por el ciclo económico. Con el objetivo de estimar una recaudación tributaria al nivel del producto potencial, es fundamental capturar el componente cíclico $h \square \square \square \square \square d e l$ ingreso tributario cuando se altera la producción nacional. 
La siguiente regresión se puede utilizar para estimar la elasticidad de los ingresos tributarios con respecto a la producción nacional:

$$
\ln \left(I T R_{i t}\right)=\alpha+\varepsilon_{1} \ln \left(P I B R_{i t}\right)+u_{i}
$$

Donde PIBR es Producto Interno Bruto de Nicaragua real a precio de referencia de 2006, ITR es el ingreso tributario en términos reales, el símbolo Ln denota el que la variable esta en logaritmo y por lo tanto el coeficiente " $\varepsilon$ " es directamente la elasticidad ingreso tributario en relación al producción nacional y " $u_{i}$ " es el componente estocástico o error de la regresión estimada.

De acuerdo al planteamiento de Bass (2006) y a lo planteado por el FMI el ingreso estructural se expresa mediante la especificación siguiente:

$$
I R T_{s, t}=I R T_{c, t}\left(\frac{Y_{t}^{\prime}}{Y_{t}}\right)^{\varepsilon}
$$

Donde $I R T_{s, t}$ representa los ingresos tributarios estructurales, $I R T_{c, t}$ representa los ingresos tributarios en términos reales, $Y_{t}$ el PIB observado, $Y_{t}^{\prime}$ el PIB potencial y " $\varepsilon$ " es la elasticidad ingreso tributario en relación al producción nacional.

También, es necesario estimar los componentes estructurales y ciclos del gasto corriente del gobierno central, la ello es necesario estimar la elasticidad del gasto corriente en relación con el Producto interno bruto(PIB), la especificación matemática es la siguiente:

$$
\ln \left(G P R_{i t}\right)=\alpha+\gamma_{1} \ln \left(P I B R_{i t}\right)+u_{i}
$$

Donde GPR es el gasto corriente del gobierno general de Nicaragua a precio de referencia de 2006, PIBR es el Producto Interno Bruto Real a precio de referencia de 2006, el término "In" denota logaritmo de la variables usadas en el modelo econométrico presentado en la ecuación (9), $\gamma_{1}$ es el coeficiente de elasticidad 
precios gasto corriente en relación con la producción nacionales el gasto corriente términos reales y " $u_{i}$ " es el error de la regresión estimada.

De acuerdo a (Basso, 2006) el gasto corriente estructural se presenta a continuación:

$$
G P R_{s, t}=G P R_{c, t}\left(\frac{Y_{t}^{\prime}}{Y_{t}}\right)^{\gamma}
$$

Donde $G P R_{s, t}$ representa el gasto corriente estructural, $G P R_{c, t}$ el gasto corriente observado a precio de referencia de 2006, el símbolo $Y_{t}$ es el PIB observado, $Y_{t}^{\prime}$ el PIB potencial y " $\gamma$ " es la elasticidad gasto corriente en relación al producción nacional.

De acuerdo a la ecuación No.6 el Balance Fiscal Estructural (BFE) para Nicaragua seria el siguiente:

$$
\text { (11) } B F E=\left(I R T_{c, t}\left(\frac{Y_{t}^{\prime}}{Y_{t}}\right)^{\varepsilon}\right)-\left(G P R_{c, t}\left(\frac{Y_{t}^{\prime}}{Y_{t}}\right)^{\gamma}\right)
$$

El periodo de análisis establecido es de 1994 a 2016, la frecuencia de los datos es anual, la fuente principal de información es el anuario estadístico del Banco Central de Nicaragua $(\mathrm{BCN})$, las variables PIB, Ingreso Tributario y Gasto Corriente se expresa en términos constante a precio de referencia de 2006, las variables fiscales fueron deflactadas con el índice deflactor del PIB, la unidad de medida de todas las variables es en millones de córdobas.

Los datos se procesaron en Microsoft Excel, el PIB Potencial (atreves del Filtro Hodrick - Prescott) y los modelos econométricos se estimaron en el programa Eviews 9. 


\section{Resultados y Análisis}

En esta sección se presenta los resultados obtenido en esta investigación sobre el balance fiscal estructural para Nicaragua y su discrepancia con los resultados de balance fiscal tradicionales registrados en las operaciones consolidadas del REICE | 123 gobierno central.

Previo a la estimación del Balance Fiscal Estructural, se estimó el PIB potencial de Nicaragua, el cual es un proxy al pleno empleo de los factores productivos en la economía.

EI PIB Potencial en Nicaragua ha evolucionado con una trayectoria similar al PIB real a precio de referencia de 2006, en la muestra evaluada se tiene registrada una brecha producto ${ }^{1}$ negativa para el año 2003 incidida principalmente por la crisis bancaria experimentada en Nicaragua en los años 2000-2001, la cual tuvo impacto en la actividad económica real dos años después.

En los años de 2009 a 2012 se registra una brecha producto negativa, la cual coincide con la crisis financiera internacional ocurrida en el año 2009, se podría inferir que resultado de la crisis internacional se generó una contracción en el pleno empleo de las actividades económicas reales en Nicaragua la cual tuvo un impacto por cuatro años y desde el año 2013 hasta 2016 la brecha producto tiene un saldo positivo la cual indica que se la producción nacional opera en un nivel superior al pleno empleo ${ }^{2}$.(Véase gráfico No.1)

\footnotetext{
${ }^{1}$ La brecha producto resulto de la diferencia del PIB Real observado y el PIB Potencial determino por el filtro Hodrick Prescott.

${ }^{2}$ El concepto de pleno empleo suelo utilizarse para referirse a una óptima utilización de factores productivos.
}

Vol. 5, No. 10, julio - diciembre 2017

REICE ISSN: 2308-782X 
Grafico No. 1 PIB Potencial y PIB Real en Nicaragua desde 1994 -2016

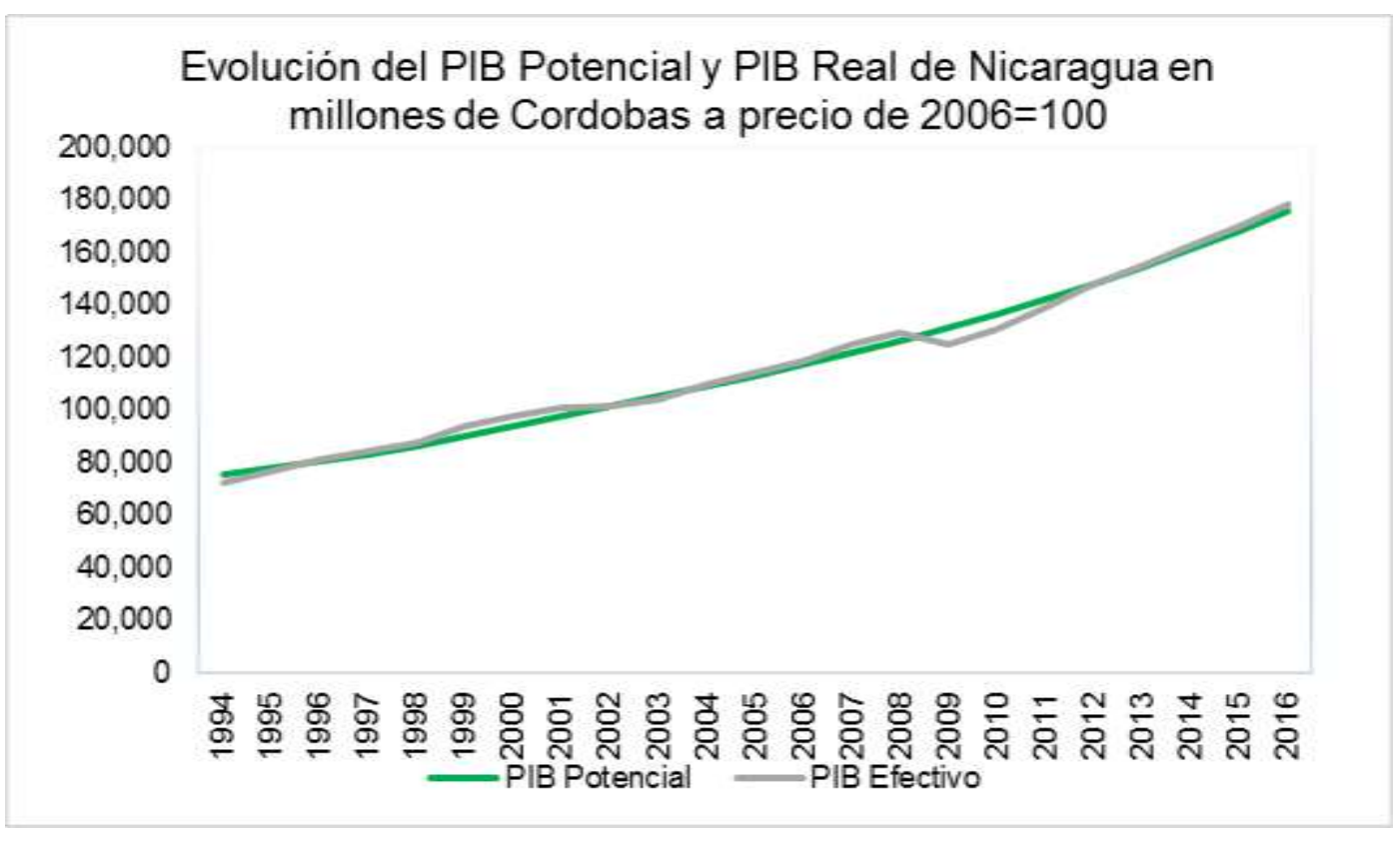

REICE | 124

Fuente: Elaboración propia con datos de BCN

El resultado del modelo econométrico propuesto en la ecuación (7) no presenta heterocedasticida, autocorrelación serial, multicolinialidad y los errores se distribuyen normalmente.

La regresión del ingreso tributario especificada en la ecuación (7) presenta una elasticidad ingreso tributario en relación de producción nacional, el valor de esta elasticidad es de 1.057263 , la cual se interpreta que por cada $1 \%$ que se incremente el PIB los ingresos tributarios se incrementaran en $1.057263 \%$, esta elasticidad es casi unitaria. (Véase anexo No.1)

Para el caso del modelo econométrico establecido en la ecuación (9), este no presenta heterocedasticida, autocorrelación serial, multicolinialidad y los errores se distribuyen normalmente.

La regresión del gasto corriente especificada en la ecuación (9) presenta una elasticidad gasto corriente en relación de producción nacional, el valor de esta de 
1.099963 la cual se interpreta que por cada $1 \%$ que se incremente el PIB el gasto corriente se incrementaran en $1.099963 \%$, esta elasticidad es ligeramente mayor a la calculada para los ingresos tributarios. (Véase anexo No.2)

Una vez estimado el PIB potencia, las elasticidades ingreso tributario y gasto corriente, se procedió a estimar las ecuaciones (8), (10) y (11) establecidas anteriormente.

En la tabla No.1 se presenta el ingreso tributario estructural y el gasto corriente estructurales, ambas variables tienen un comportamiento similar a los datos contabilizados por el en gobierno central.

Tanto la variable ingreso fiscal estructural y gasto corriente estructural, presentan una trayectoria similar al PIB potencial estimado, es decir, al comportamiento del ciclo económico.

En los años 2003, 2009,2010,2011 y 2012 se presenta valores observados para ingreso tributario y gasto corriente menores a los saldos de estas mismas variables en su versión estructural, es significativo que es eso mismo año se registra una brecha producto negativa, por lo que puede inferir que las decisiones de política fiscal tienen un componente discrecional y otro incidido por el ciclo económico. (Véase Tabla No. 1) 
Tabla No.1 Comparación de los Ingreso tributario, Gasto Corriente, con los Ingreso Tributarios Estructurales y el Gasto Corriente Estructural. (En millones de Córdobas a precio de 2006)

\begin{tabular}{|c|c|c|c|c|c|c|}
\hline Años & $\begin{array}{l}\text { Ingreso } \\
\text { Tributario }\end{array}$ & $\begin{array}{l}\text { Ingreso Tributario } \\
\text { Estructural }\end{array}$ & $\begin{array}{l}\text { Variación } \\
\text { Porcentual }\end{array}$ & $\begin{array}{l}\text { Gasto } \\
\text { Corriente }\end{array}$ & $\begin{array}{c}\text { Gasto } \\
\text { Corriente } \\
\text { Estructural }\end{array}$ & $\begin{array}{l}\text { Variación } \\
\text { Porcentual }\end{array}$ \\
\hline 1994 & 10,496 & 11,029 & -5.08 & 11,428 & 12,034 & -5.29 \\
\hline 1995 & 11,387 & 11,584 & -1.73 & 10,662 & 10,854 & -1.80 \\
\hline 1996 & 12,150 & 11,982 & 1.39 & 11,794 & 11,625 & 1.44 \\
\hline 1997 & 13,902 & 13,666 & 1.70 & 12,315 & 12,098 & 1.76 \\
\hline 1998 & 15,259 & 15,030 & 1.50 & 13,526 & 13,315 & 1.56 \\
\hline 1999 & 16,111 & 15,399 & 4.42 & 14,029 & 13,385 & 4.59 \\
\hline 2000 & 17,085 & 16,322 & 4.47 & 15,907 & 15,169 & 4.64 \\
\hline 2001 & 13,448 & 12,984 & 3.45 & 16,573 & 15,979 & 3.58 \\
\hline 2002 & 14,348 & 14,315 & 0.23 & 15,617 & 15,580 & 0.24 \\
\hline 2003 & 16,565 & 16,756 & -1.15 & 16,816 & 17,018 & -1.20 \\
\hline 2004 & 18,168 & 18,101 & 0.37 & 16,309 & 16,246 & 0.38 \\
\hline 2005 & 20,017 & 19,840 & 0.88 & 17,775 & 17,611 & 0.92 \\
\hline 2006 & 17,057 & 16,834 & 1.30 & 16,001 & 15,784 & 1.36 \\
\hline 2007 & 18,214 & 17,730 & 2.66 & 16,210 & 15,762 & 2.76 \\
\hline 2008 & 17,905 & 17,480 & 2.38 & 18,377 & 17,923 & 2.47 \\
\hline 2009 & 17,198 & 18,091 & -5.19 & 18,983 & 20,009 & -5.41 \\
\hline 2010 & 18,678 & 19,551 & -4.67 & 19,439 & 20,385 & -4.86 \\
\hline 2011 & 21,040 & 21,536 & -2.36 & 20,719 & 21,227 & -2.45 \\
\hline 2012 & 23,205 & 23,222 & -0.07 & 22,916 & 22,933 & -0.08 \\
\hline 2013 & 24,423 & 24,301 & 0.50 & 24,365 & 24,239 & 0.52 \\
\hline 2014 & 26,059 & 25,838 & 0.85 & 26,802 & 26,565 & 0.88 \\
\hline 2015 & 27,829 & 27,489 & 1.22 & 28,518 & 28,155 & 1.27 \\
\hline 2016 & 30,308 & 29,874 & 1.43 & 32,101 & 31,622 & 1.49 \\
\hline
\end{tabular}

Fuente: Elaboración propia con datos de BCN

Los resultados indican que se presenta un componente estructural en la política fiscal muy relevante en Nicaragua, a razón que el balance primario convencional y el balance primario estructural tiene diferencias mínimas, con estos resultados se puede inferir que el componente de gasto corriente tiene un comportamiento similar a la trayectoria de la producción nacional, es decir, que sigue un patrón pro-cíclico. (Véase Gráfico No. 2) 
Grafico No. 2 Balance Primario Convencional y Balance Primario Estructural en Porcentaje del PIB Real y PIB potencial respectivamente en Nicaragua.

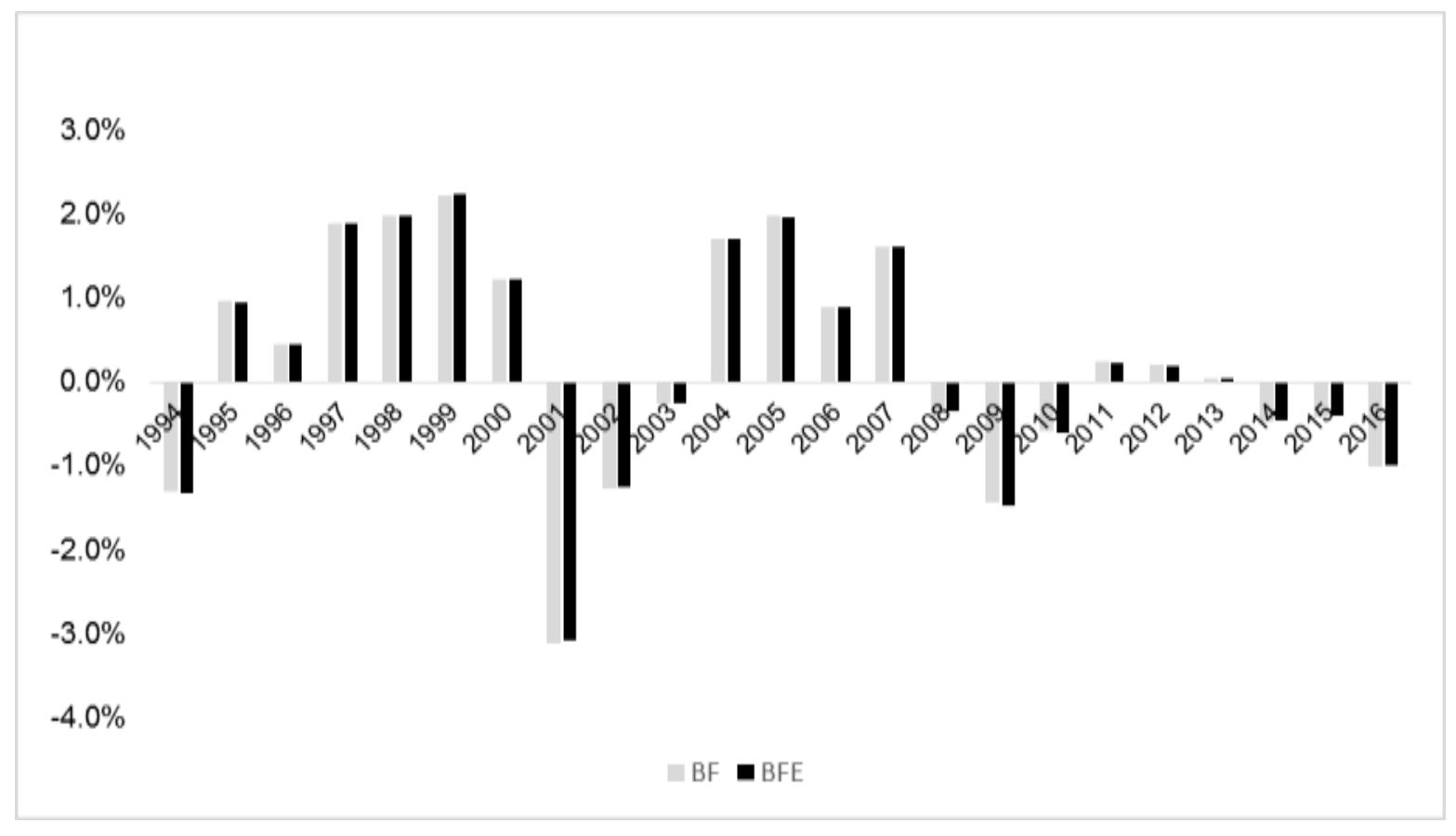

Fuente: Elaboración propia con datos de BCN

Se puede evidenciar en el gráfico No.2 que desde 1994 hasta 2016, el balance fiscal convencional y balance fiscal estructural ha tenido un resultado errático, sin una tendencia clara y definida de déficit o de superávit.

En todos los años evaluados, tanto para saldo de déficit como de superávit en el componente estructural y convencional es marginalmente diferente. En los años 1994,2001, 2002, 2003, 2008,2009 y 2010 se presenta un déficit estructural ligeramente menor al déficit convencional este resultado coincide con una brecha producto negativa.

El en el año 2001 se registró el déficit fiscal convencional más alto, debido principalmente a un aumento del $47 \%$ en las transferencias corriente del gobierno, también de un crecimiento $37.1 \%$ en el pago de interés, de un crecimiento de $11.5 \%$ en sueldo y salarios públicos y un crecimiento de $9.5 \%$ en la compra de bienes y servicios. 
Luego en los años 2009 y 2016 se presenta un déficit convencional muy alto, incidido principalmente un alto crecimiento en las partidas de gasto transferencias corriente, sueldos y salarios públicos y consumo de bienes y servicios.

Al observar el comportamiento de las variaciones porcentuales del PIB real, los ingresos y los gastos totales registrados por el BCN, se pueden corroborar los resultados del modelo de Balance Estructural Fiscal implementado en el presente estudio.

\section{Grafica No. 3 Variacion Interanual del PIB real, Ingresos y Gastos totales reales. \\ Perido 1994 - 2016}

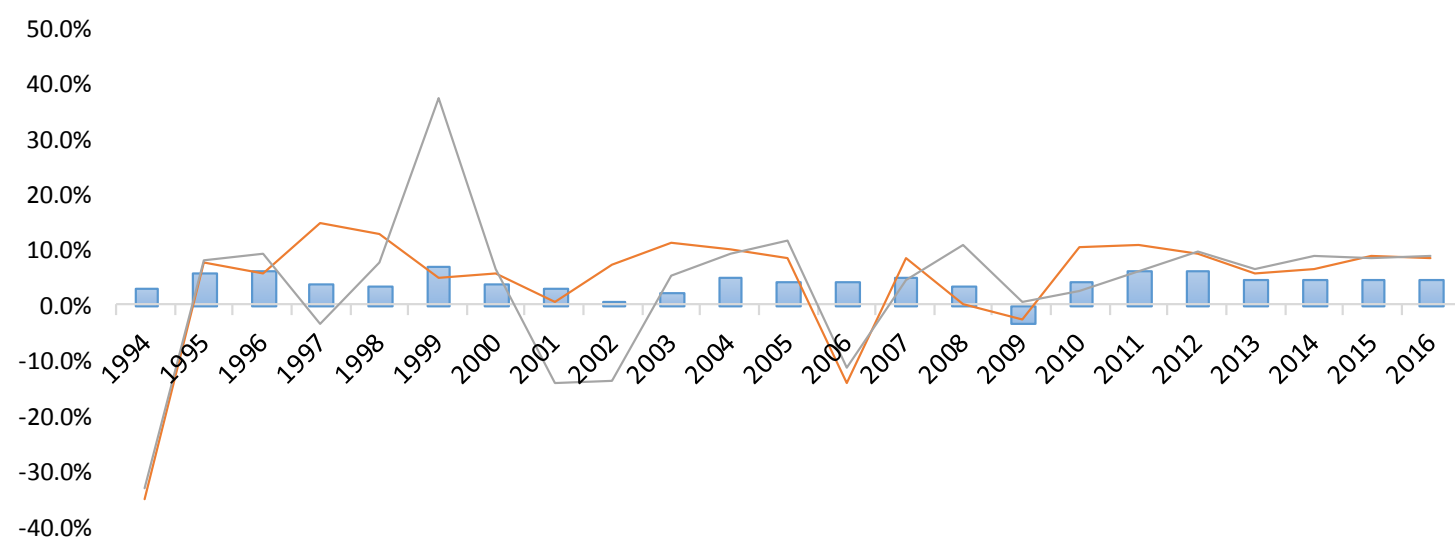

$\square$ PIB 100=2006 - Ingresos Total Real - Gastos Total Real

Fuente: Elaboracion propia con datos del bcn

En el grafico No. 3, se observa una correlación entre el PIB real y el gasto total, quedando en evidencia una actividad pro-cíclica de la política fiscal. En los periodos en donde la producción se aceleró de igual manera se manifestó un incremento del gasto, esto ocurre también en efectos contrarios del comportamiento del PIB.

Para el año de 2002, 2006 y 2009, se manifestó una disminución del PIB en su crecimiento, de igual forma, para estos mismos años, el gasto total también experimento una desaceleración y disminuyo significativamente. Sin embargo, para los años de 1991, 2004 y 2012, el gasto total experimento un incremento, donde también se reflejó igual comportamiento en la producción real. 


\section{Conclusiones}

En este artículo se estimó el balance fiscal estructural para Nicaragua, para ello se utilizó la metodología propuesta por Fondo Monetario Internacional (FMI). A partir de los resultados analizado se pueden inferir las siguientes conclusiones.

Los indicadores de ingreso fiscal estructural y Gasto corriente estructural estimados para Nicaragua exponen los resultados de las medidas de política fiscal discrecional usado por el gobierno y que el déficit fiscal emana de un comportamiento estructural que puede ser usado para efectuar correcciones en los resultados del balance consolidados de las finanzas públicas, estos resultados se presentan únicamente con el uso indicadores estructurales especificados en la ecuación (8), (9) y (11), estos resultados son similares a los encontrados por (Rojas, 2017)

Desde 1994 hasta 2016 el balance fiscal estructural tiene el mismo comportamiento que el balance fiscal convencional registrado en sistema de cuentas nacionales de Nicaragua y también tiene el mismo sentido que comportamiento que la brecha producto.

La metodología usada en este artículo para la determinación del balance estructural fiscal contribuye a la literatura econométrica de las finanzas públicas nicaragüenses, y los resultados puede ser útil para establecimiento de los modelos macrofiscales o para el desarrollo de una regla fiscal en Nicaragua. esto temas se ubican en agenda de investigación.

Es importante mencionar que existen diferentes metodologías para estimar el balance fiscal estructural, y los resultados de otras metodologías puede diferir de los estimados en este documento, para un detalle más completo revisar (Basto Mercados , 2003).

La elasticidad ingreso en relación a la producción nacional estimada es de un valor de $1.057263 \%$, esta se puede caracterizar como elasticidad unitaria. Ahora bien, la elasticidad gasto corriente en relación al PIB real estimo un valor de $1.099963 \%$, esta es relativamente más baja que la elasticidad ingreso producto. 
EI PIB potencial estimado con el filtro Hodrick- Prescott, utilizo una landa de 100 como parámetro de suavizamiento del PIB real, resultados bajo distintos datos puede diferir mucho, producto que en la literatura econométrica existen varias metodologías que también se usan para estimar el PIB potencial, por lo tanto, el resultado no se debe de considerar como único y definitivo.

REICE | 130

En este mismo sentido, el resultado del estudio deja en manifiesto que la política fiscal nicaragüense es pro-cíclica. Esto debido a la correlación que existe entre el gasto y el PIB real, en los periodos que las la producción tiene un auge el gasto se expande, y de forma contraria, cuando existe una disminución de la producción el gasto total se contrario.

\section{Bibliografía}

Basso, M. (2006). El Balance Estructural: Metodologias y Estimación Para Argentina. Analisis de Asociación Argentina de Economía Politica, 3-15.

Basto Mercados , L. E. (2003). Metodologías de estimación del balance estructural:: Bogota Colombia: Departamento Nacional de Planeacion: Direccion de Estudios Economicos .

Beteta, H. (2012). El balance fiscal estructural en Centroamerica y Republica Dominicana . Finanzas Publicas en America Latina, 6.

Hagémann, R. (1999). The Structural Budget Balance The IMF's Methodology (IMF Working Paper No 99/95). International Monetary Fund, 2-15.

Price, R. (2011). Adjusting Fiscal Balances for Asset Price Cycles . OECD Economics Department Working Papers No. 868, 2.

Rojas, D. (2017). Balance Estructural Fiscal en Costa Rica 1998-2014. Revista de Ciencias Economicas de la Universidad de Costa Rica, 1-17.

Segura Rodríguez, C., \& Vásquez Carvajal, J. (2011). Estimación del parámetro de suavizamiento del filtro de Hodrick y Prescott para Costa Rica. Banco Central de Costa Ricas, 3- 5. 
Anexos 1 Estimación de la elasticidad ingreso Producto para Nicaragua.

Dependent Variable: LNITR

Method: ARMA Maximum Likelihood (BFGS)

Date: 12/02/17 Time: 06:32

Sample: 19942016

Included observations: 23

REICE | 131

Convergence achieved after 3 iterations

Coefficient covariance computed using outer product of gradients

\begin{tabular}{crrrr}
\hline \multicolumn{1}{c}{ Variable } & Coefficient & Std. Error & t-Statistic & Prob. \\
\hline \hline C & -2.530615 & 2.202451 & -1.148999 & 0.2648 \\
ANPIBR & 1.057263 & 0.189629 & 5.575417 & 0.0000 \\
SIGMASQ & 0.475310 & 0.246968 & 1.924582 & 0.0694 \\
\hline \hline R-squared & 0.005217 & 0.002079 & 2.508841 & 0.0213 \\
Adjusted R-squared & 0.929235 & Mean dependent var & 9.777400 \\
S.E. of regression & 0.918062 & S.D. dependent var & 0.277623 \\
Sum squared resid & 0.079469 & Akaike info criterion & -2.058987 \\
Log likelihood & 0.119992 & Schwarz criterion & -1.861510 \\
F-statistic & 27.67836 & Hannan-Quinn criter. & -2.009322 \\
Prob(F-statistic) & 83.16485 & Durbin-Watson stat & 1.718167 \\
\hline \hline Inverted AR Roots & 0.000000 & & \\
\hline \hline
\end{tabular}

Prueba de Heterocedasticidad de la Función Ingreso Tributario.

Heteroskedasticity Test: Breusch-Pagan-Godfrey

\begin{tabular}{llll}
\hline \hline F-statistic & 0.530288 & Prob. F $(1,21)$ & 0.4745 \\
Obs*R-squared & 0.566487 & Prob. Chi-Square(1) & 0.4517 \\
Scaled explained SS & 0.550586 & Prob. Chi-Square(1) & 0.4581 \\
\hline \hline
\end{tabular}

Test Equation:

Dependent Variable: RESID^2

Method: Least Squares

Date: 12/03/17 Time: 10:00

Sample: 19942016

Included observations: 23

\begin{tabular}{lrllr}
\hline \hline \multicolumn{1}{c}{ Variable } & Coefficient & Std. Error & t-Statistic & Prob. \\
\hline \multicolumn{1}{c}{ C } & 0.068654 & 0.087134 & 0.787910 & 0.4396 \\
\multicolumn{1}{c}{ LNPIBR } & -0.005449 & 0.007482 & -0.728209 & 0.4745 \\
\hline \hline R-squared & 0.024630 & Mean dependent var & 0.005217 \\
Adjusted R-squared & -0.021816 & S.D. dependent var & 0.009003 \\
S.E. of regression & 0.009101 & Akaike info criterion & -6.478010 \\
Sum squared resid & 0.001739 & Schwarz criterion & -6.379272 \\
Log likelihood & 76.49712 & Hannan-Quinn criter. & -6.453178 \\
F-statistic & 0.530288 & Durbin-Watson stat & 2.074443 \\
Prob(F-statistic) & 0.474528 & & \\
\hline \hline
\end{tabular}

Vol. 5, No. 10, julio - diciembre 2017

REICE ISSN: 2308-782X 
Anexos 2 Estimación de la elasticidad Gasto Corriente en relación con el Producto para Nicaragua.

Dependent Variable: LNGPR

Method: ARMA Maximum Likelihood (BFGS)

Date: 12/02/17 Time: 06:39

REICE | 132

Sample: 19942016

Included observations: 23

Convergence achieved after 4 iterations

Coefficient covariance computed using outer product of gradients

\begin{tabular}{crlrr}
\hline \hline \multicolumn{1}{c}{ Variable } & Coefficient & Std. Error & t-Statistic & Prob. \\
\hline C & -3.032223 & 1.125120 & -2.695021 & 0.0143 \\
LNPIBR & 1.099963 & 0.096796 & 11.36371 & 0.0000 \\
AR(1) & 0.570152 & 0.191705 & 2.974106 & 0.0078 \\
SIGMASQ & 0.003310 & 0.001128 & 2.933869 & 0.0085 \\
\hline \hline R-squared & 0.959620 & Mean dependent var & 9.763659 \\
Adjusted R-squared & 0.953244 & S.D. dependent var & 0.292744 \\
S.E. of regression & 0.063300 & Akaike info criterion & -2.507986 \\
Sum squared resid & 0.076132 & Schwarz criterion & -2.310509 \\
Log likelihood & 32.84184 & Hannan-Quinn criter. & -2.458321 \\
F-statistic & 150.5094 & Durbin-Watson stat & 1.927713 \\
Prob(F-statistic) & 0.000000 & & & \\
\hline \hline & & & & \\
Inverted AR Roots & .57 & & & \\
\hline \hline
\end{tabular}

Prueba de Heterocedasticidad para la Función Gasto Corriente.

Heteroskedasticity Test: Breusch-Pagan-Godfrey

\begin{tabular}{llll}
\hline \hline F-statistic & 0.067150 & Prob. F(1,21) & 0.7981 \\
Obs*R-squared & 0.073311 & Prob. Chi-Square(1) & 0.7866 \\
Scaled explained SS & 0.055068 & Prob. Chi-Square(1) & 0.8145 \\
\hline \hline
\end{tabular}

Test Equation:

Dependent Variable: RESID^2

Method: Least Squares

Date: 12/03/17 Time: 10:02

Sample: 19942016

Included observations: 23

\begin{tabular}{lrlrr}
\hline \hline \multicolumn{1}{c}{ Variable } & Coefficient & Std. Error & t-Statistic & Prob. \\
\hline \multicolumn{1}{c}{ C } & 0.016039 & 0.049132 & 0.326443 & 0.7473 \\
\multicolumn{1}{c}{ LNPIBR } & -0.001093 & 0.004219 & -0.259134 & 0.7981 \\
\hline \hline R-squared & 0.003187 & Mean dependent var & 0.003310 \\
Adjusted R-squared & -0.044280 & S.D. dependent var & 0.005022 \\
S.E. of regression & 0.005132 & Akaike info criterion & -7.623860 \\
Sum squared resid & 0.000553 & Schwarz criterion & -7.525121 \\
Log likelihood & 89.67439 & Hannan-Quinn criter. & -7.599027 \\
F-statistic & 0.067150 & Durbin-Watson stat & 1.667105 \\
Prob(F-statistic) & 0.798055 & & \\
\hline \hline
\end{tabular}

